DOI: https://doi.org/10.3897/arb.v33.e09

\title{
MATHEMATIC- SYSTEMIC RESEARCHING WITH THE INVOLVEMENT OF FRACTALS WHEN PROCESSING OF SPACE SURVEILLANCE SYSTEMS ON RECOGNITION OF WASTE DISPOSAL OBJECTS
}

\author{
Maretta Kazaryan \\ North Ossetian State Medical Academy \\ e-mail:marettak@bk.ru
}

Keywords: Waste Disposal Objects, Satellite Imagery Data, Involvement of Fractals

\begin{abstract}
The article analyzes the factors that stimulate research and development of an automated system for processing and analyzing satellite imagery data. In particular, the study of satellite imagery for the presence of waste disposal objects is considered as an application. This problem is of particular interest when conducting scientific research. The development of automated control systems (ACS) in the field of space image (SI) recognition is characterized primarily by the approach of interest, i.e. for what purpose and for solving of which particular problem of the subject area the system is created. Obviously, the system is a set of certain information technologies and the main factor in the development of an automated system is and remains the study of peculiarities of creating an appropriate information system (IS). The article proposes a methodological approach to the processing and analysis of data by the IS. The approach is based on the conceptual idea of fractal sets. This enhances the detection of abnormal signals in difficult phono-target environments. Testing is conducted on the detection of WDS under conditions of a low signal-to-noise ratio.
\end{abstract}

\section{Introduction}

Modern realities require a new approach for the presence of certain problems when monitoring that are directly related to the directions of development of the national economy of our country. This includes hydrometeorological conditions, ecological conditions, frequent forest fires, etc. With the advent of open global networks, satellite information has become more accessible to scientists, researchers and workers in all areas of the country's economy. The works [1-3, 26-27] ware devoted to this issue. Namely, the need to create an information system for monitoring territories for a certain range of tasks, in our case, we investigate the presence of the waste disposal facilities (WDF) and industrial waste (IW). 
The article discusses the problems of waste disposal facilities and the creation of monitoring systems, carrying out the system-mathematical aspect of the problem under study. Issues related to the architecture of building an information system that monitors territories for the presence of WDF, were discussed in articles [26-27]. This paper continues the study of this problem.

The problem of identifying unauthorized landfills and monitoring the correct operation of the existing landfills in accordance with the current regulatory documents is actual, and the solution to this problem would be very relevant in order to draw attention to the issues of environmental development and biodiversity conservation. In his interview "On the rubbish disaster of Russia", Russian President Vladimir Putin acknowledged that Russia does not have a complete waste recycling system, there is no separate waste collection system, this could face an environmental catastrophe for some areas. He noted that only half of all waste in Russia is disposed of, the rest is buried. "And this process is in most cases uncontrolled and criminalized," he said (https://lenta.ru/news/2016/04/14/musor/).

This problem is of interest from a scientific point of view. The development of automated control systems (ACS) in the field of space image (SI) recognition is characterized primarily by the approach of interest, i.e. for what purpose and for solving of which particular problem of the subject area the system is created. Obviously, the system is a set of certain information technologies and the main factor in the development of an automated system is and remains the study of features when creating an appropriate information system (IS).

Using the example of processing and recognition of a SI for the presence of an WDF with a small signal-to-noise ratio, we will conduct some mathematical research and in support of the obtained results we will conduct an experiment or approbation.

\section{The research part}

One of the leading directions in the analysis of aerospace monitoring data is the development of the theory of digital multispectral and hyperspectral images of the Earth and the atmospheric surface layer processing to solve the problem of automatic (without human participation) recognition of useful signals localized in time and space randomly in the background of additive and multiplicative noise.

First of all, the study of the task of deciphering of SI is the analysis of images and of applied tasks. The image is a multi-dimensional signal. The basis of information technology (IT) of image processing includes mathematical principles, the nature of which is determined by the range of tasks. The following studies are known in the field of image processing for the creation of IS and their mathematical analysis in certain areas. Thus, on the problems of integrating system-wide approaches to image analysis [13], on developing mathematical 
models of images that have a stochastic nature [14], on image analysis using an adaptive filtering method [15], on recovering signals based on regularization of inverse problems [16], the synthesis of regenerative filters for the case of a small number of observations [17], by statistical texture analysis of images [18], by automatic classification and recognition of images based on statistical clustering [19], by algebraic by the traditional methods of image analysis in the form of a set of primitives having a geometric shape [20], by non-parametric image analysis methods [21], by neural network image processing technologies [22], by system structural analysis based on Peano-Hilbert type quasi-continuous sweeps [23], by system structural analysis based on field analysis having a structurally redundant quasiperiodic character [24], according to system structural analysis based on fractal signatures [25].

Analyzing the problem of detecting and evaluating signals of a random nature, it is possible to identify factors that contribute to the creation of an automated geo-ecological system for detecting WDF. The brightness fields form the background in the space monitoring task. The first factor can be formulated as follows: the background is non-stationary in the one-dimensional and non-uniform in the n-dimensional case. The second factor - the brightness fields have the property of non-Gaussianity. The third factor - the background environment is a non-Markov process. Consequently, it is necessary to take into account the correlation of long-range order, limiting the use of classical correlation methods. The fourth factor - the background is a singular process, i.e. it is non-differentiable and this causes mathematical difficulties in data processing. The fifth factor is that the signal-to-noise ratio is rather small and this leads to certain problems in signal detection and evaluation. The sixth factor is the prior uncertainty of signal knowledge, i.e. form uncertainty. The seventh factor - the spatial-temporal resolution of the means of observation is rather limited.

So, the basis of the developed information system for the processing of satellite imagery is the creation of a special information technology (IT) to extract information about WDS from the space image.

The theoretical and conceptual approach to this problem can be characterized as follows. The core of the space observation systems (SOS) for the recognition of WDS is the integration of certain concepts that are directly related to the concept of multi-scale representation of signals. In system integration, all concepts have a certain dominant role. One of those concepts is the study of fractal sets. The dominant function in this concept is the simulation of phono-target environment based on large-scale self-similarity. Further research will be devoted to the study of the remaining concepts that have a role in the formation of IT, used for thematic interpretation of the SI as part of an automated information system.

The mathematical model of the information processing system for information processing in the monitoring of territories for the presence of WDS is a stochastic process with a fractal structure. 
Why namely the theory of fractals attracts our attention. As is known [2], when modeling the space image of territories, it is necessary to investigate the physical processes of dispersion, radiation of electromagnetic waves from the surface, in our case, the WDS.

If to consider the statistical characteristics of the WDS, then, as a rule, they are determined by the statistical characteristics of the irregularities of the surfaces that make up the WDS. In any WDS surface irregularities are connected and formed under the influence of certain factors - thermal, chemical, etc. There are different methods and approaches for describing the scattering and radiating surfaces of the objects under study. You can consider single-scale irregularities using a single-scale correlation radius and, accordingly, multiscale irregularities using a multi-scale correlation radius [4-8]. With an increase in the order of multiscale, the multi-scale of the correlation radius also increases and this leads to the complication of the description of the mathematical model of the investigated space image. That is why it makes sense to pass to the fractal representation of the image, specifically the space image in the monitoring of territories, the presence of the WDS. Let us take preliminary studies related to the theory of fractals.

According to the theory of fractals [4-8], any natural object has a fractal self-similar organization structure. Each image has a scan - let's denote it by $\xi(x)$.

Definition 1. Let there be a set of hierarchical levels $i$. Each level is represented by a certain scale and radius of correlation. Then the superposition of these levels is defined as follows:

$$
\xi(x)=\sum_{i=1}^{\infty} \xi_{i}(x)
$$

Definition 2. Each level $i$ have a statistical ordering defined by a correlation function $\left\langle\xi_{i}\left(x_{1}\right) \xi_{i}\left(x_{2}\right)\right\rangle$.

Let us assume that the correlation of this level is Gaussian, i.e.

$$
\left\langle\xi_{i}\left(x_{1}\right) \xi_{i}\left(x_{2}\right)\right\rangle=\sigma_{i} \exp \left[-\frac{\left(x_{1}-x_{2}\right)^{2}}{\rho_{i}^{2}}\right]
$$

Here $\sigma_{i}$ is the variance, $\rho_{i}$ is the correlation radius. Each component $\xi_{i}$ (x) has a certain zone of influence, the scale of which is given by the radius of correlation $-\rho_{i}$.

Let us suppose that the quantities $\sigma_{i}$ and $\rho_{i}$ obey scaling laws - this follows from the scale invariance of multilevel relaxation processes, i.e.

$$
\sigma_{k}=\frac{\sigma_{0}}{a^{k}} \text { and } \rho_{k}=\rho_{0} \cdot b^{k}
$$


For hierarchically organized processes the delimitation of zones of influence at different levels, is characteristic, i.e. there is a relationship: $\rho_{i-1}<<\rho_{i}<<\rho_{i+1}$

Independence of different-scale correlations follows from this relation:

$$
\left\langle\xi_{i}\left(x_{1}\right) \xi_{i}\left(x_{2}\right)\right\rangle=0, \quad i \neq j
$$

From here, the correlation function will have the following form:

$$
\left\langle\xi_{i}\left(x_{1}\right) \xi_{i}\left(x_{2}\right)\right\rangle=\sigma_{i} \exp \left[-\frac{\left(x_{1}-x_{2}\right)^{2}}{\rho_{i}^{2}}\right]
$$

Taking into account the scaling properties of $\sigma_{i}$ and $\rho_{i}$ the parameters and we will have:

$$
\left\langle\xi_{i}\left(x_{1}\right) \xi_{i}\left(x_{2}\right)\right\rangle \cong \sigma_{0} \int_{0}^{\infty} \exp (-x p) \exp \left[-\frac{\left(x_{1}-x_{2}\right)^{2}}{\rho_{i}^{2}} \exp (-x y)\right] d x
$$

Let's give the asymptotic estimate of this integral with $x_{1}-x_{2}=\rho \rightarrow \infty$ :

$$
\begin{aligned}
& \left\langle\xi_{i}\left(x_{1}\right) \xi_{i}\left(x_{2}\right)\right\rangle_{\left|x_{1}-x_{2}\right| \rightarrow \infty} \approx\left(\frac{\rho}{\rho_{0}}\right)^{-\alpha} \\
& \text { here - } \alpha=\ln a / \ln b .
\end{aligned}
$$

Let's consider as a structural object (set) the totality of brightness, forming a row (scan) of the satellite image when scanning the earth surface by the satellite of space observation ( $\mathrm{SSO}$ ).

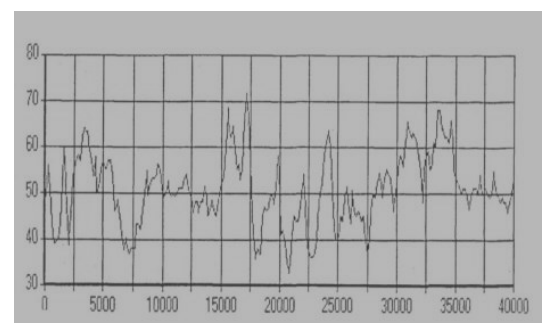

a) MSU-SK data $R=160 \mathrm{~m}$

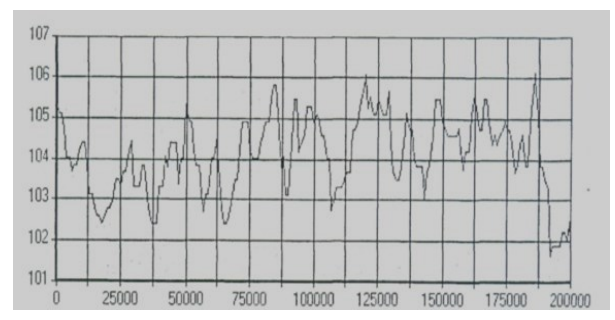

b) NOAA data, AVHRR, $R=1100 \mathrm{~m}$ 


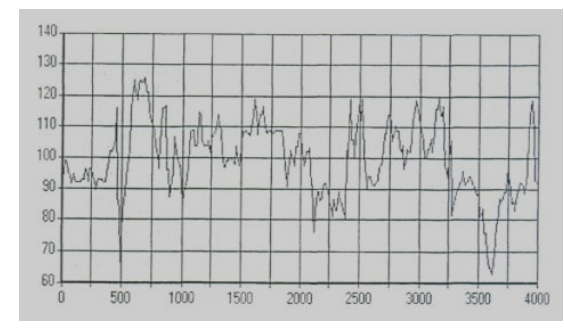

c) SPOT data, VHR, $R=10 \mathrm{~m}$

Fig. 1. Scanning of space images as structural objects with fractal properties

Fig. 1 gives a set of brightness, which together form an irregular curve associated with a random process. The scan of the image line in Figure 1 (b) is the spatial resolution of the observation system $R=1100 \mathrm{~m}$., i.e. - this is a discrete sampling with an interval of $1100 \mathrm{~m}$, obtained by the AVHRR scanner of the NOAA satellite system. Fig. 1 (c) - scan of the image line from the SPH satellite based on VHR, spatial resolution $R=10 \mathrm{~m}$, i.e. we have a sample at intervals of $10 \mathrm{~m}$.

From Fig. 1 it is clear that the sample scale does not change the appearance of the curves. An image scan is a structural object that, in a mathematical sense, can be represented as a random process with the property of scale self-similarity.

So, when developing a mathematical model of a space image, we will proceed from the fact that a space image is a stochastic process with a fractal structure [3].

Why exactly the theory of fractals is so interesting for us when building a mathematical model of a satellite image (SI) - once again we'll dwell on this issue as applied to the WDS. As is known [2-3], when modeling these image data, it is necessary to investigate the physical processes - scattering, radiation of electromagnetic waves from the surface, in our case, WDS.

If we consider the statistical characteristics of the WDS, then they, as a rule, are determined by the statistical characteristics of the irregularities of those surfaces that are part of the WDS.

In a WDS, surface irregularities are connected and formed under the influence of certain factors - thermal, chemical, etc. [2].

There are different methods and approaches for the description of the scattering and radiating surfaces of the investigated objects of a WDS. You can consider single-scale irregularities using a single-scale correlation radius and multiscale irregularities using a multi-scale correlation radius [4-7]. With an increase in the order of multi-scale, the multi-scale of the correlation radius increases and this leads to the complication of the description of the mathematical 
model of the given space image. Therefore, it is advisable to go to the fractal representation of the image, specifically the space image, while monitoring for the presence of a WDS.

Any natural object has a fractally self-similar organization structure [4]. As is known [3-4], a texture is a matrix or a fragment of the spatial properties of sections of images with uniform statistical characteristics. The method of describing image textures is the relationship with the spatial distributions and interdependence of the brightness values of the local area of the image or block. Textural signs refer to probabilistic signs. Random values of textural attributes are distributed to all classes of natural objects. Therefore, the concept of a texture signature is introduced. A texture signature is the distribution of the total population of measurements for a given texture in scenes of the same type as this one.

Now let's turn to the concept of fractal. The fractal dimension characterizes the degree of filling of the space in which the fractal system exists. The theory of fractals considers fractional instead of integer measures and uses new quantitative indicators, namely the fractional dimensions of $D$ and the corresponding fractal signatures $[4,9-10,12]$. The theory of fractals and nonlinearity constitute the geometry of chaos. The contours of all natural objects are dynamic processes, frozen in physical forms and combining chaos and stability.

One of the most important issues of fractal geometry is the relationship of fractals and textures and this can be successfully applied in the tasks of detecting and recognizing low-contrast targets against the background of earthly covers.

According to Mandelbrot [4]: "A fractal is a functional mapping or a set obtained by an infinite recursive process and having the following properties 1) self-similarity, 2) their dimension (Hausdorff dimension) is fractional and strictly more than topological dimension, 3) non-differentiable and operating with fractional derivatives and integrals.

Fractal processing of low-contrast images is an unconventional way to solve a similar problem.

The establishment of the invariance of the fractal dimensions of $D$ images from natural formations, in this case the WDS; of their brightness is the main prerequisite for the transition to fractal processing of low-contrast images.

The main idea of further research is as follows. Any deterministic object in certain territorial limits of observation has the same characteristics - size and area. When you change the scale of the terrain image, the area of fractal formations also changes. The appearance of an artificial object on any image changes the fractal dimension $D$ of the complex image as a whole. image $A^{\prime}$.

The scale can be varied by frame filtering the source image $A$ into

Let $f_{i j}$ be elements of the image $f_{H}(x, y)$ of size $N x \times N y$., which is subjected to frame filtering, $f^{\prime}{ }_{m n}-$ the elements of the resulting image $f_{H}{ }_{H}(x, y)$ of 
size $N x \times N y$. Then for filtering with a frame or a window $(2 M+1)(2 N+1)$ elements, we have:

$$
A \rightarrow A^{\prime}: f_{m n}^{\prime} / \forall i j=\frac{1}{(2 M+1)(2 N+1)} \sum_{i=m-M}^{m+M} \sum_{j=n-N}^{n+N} f_{i j}
$$

The objective presence of fractional dimensionality and scale invariance in fractals makes it possible to investigate the problem of detecting a WDS from a new point of view for small signal-to-noise ratios [5-7].

The article presents the initial experimental studies on this topic on a WDS.

The purpose of the study is the possibility of analyzing optical observation systems, as a result of which we have a SI to determine a WDS using, and constructing the field of fractal dimensions (FFD) and choosing the parameters for constructing FFD.

\section{Experimental part} algorithm:

The construction of the FFD is carried out according to the following

- image scanning by "window" with the following parameters: window size $a \times b$ pixels, movement step $s$ (for $s=1$ "window" sliding, for $s>1$ window jumping);

- at each step, the numerical value of the dimension $D_{i, j}$ in the "window" is determined and written into the matrix $D$. This matrix will be called the "field of fractal dimensions".

The formula for calculating the number of rows and columns of a matrix depends on the number of rows and columns of the analyzed image.

$M, N$ - the number of rows and columns of the matrix $D$;

$m, n$ - the number of rows and columns of the analyzed image;

$a$ (b) - the size of the "window";

$s$ - the value of the "jump" "window";

] [ - procedure for taking the whole part.

$$
M(N)=] \frac{m(n)-a(b)}{s}[+1
$$

The image of the WDS at the SI is considered as the initial information allocated for the study. The WDS is displayed on the earth's surface, namely, the Kuchino SW landfill (August 2011) is given; similarly, you can further consider the object of household waste on the water surface $[2,11]$. 


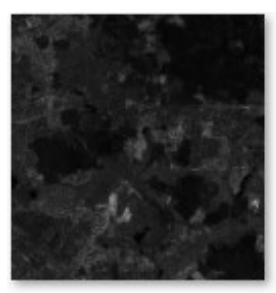

$a$

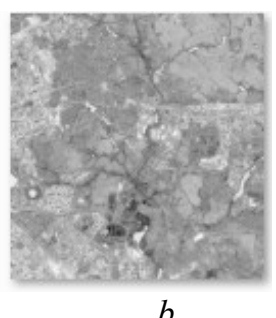

$b$

Fig. 2: $a$ - the image on the SI has a size $270 \times 270$.); $b$ - field of fractals dimensions $(270 \times 270$ pixels $)$

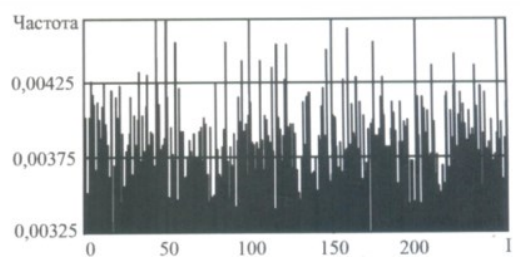

Fig. 3. Histogram of brightness

It is assumed that no pre-processing is carried out with the original image, i.e. we want to test the fractal method of processing the original signal. Let's consider the basic parameters of the field of fractal dimension. A test image is an image whose brightness has a uniform distribution law in the range from 0 to 1 and their fractal dimension $D \approx 2,5$.

Step 1: generation of the field of fractal dimensions. Figure 2 (b) gives the field of fractal dimensions, constructed using the "sliding window" of $17 \times 17$ pixels. To calculate the fractal dimension is used - the prism method [8-10]. A visual analysis of the FFD of the original image shows that the surface along the river has some features that are not visible on the SI. The type and speed of building a FFD are influenced by the size of the "window" and the displacement step. The test image is given in Figure 2 (a). The areas of location of natural objects on the fractal binary image have zero brightness, and the areas of location of WDS - maximum brightness. The total number of bright points of the object specified by the binary image is stable and obtained experimentally from the reference image of the object. To detect all areas of the image, which assumes the presence of WDS, you must scan it using a sliding window; the window size corresponds to the size of the object.

Step 2: Construction of a histogram of distribution $I$ in the interval $[0,255]$.

Step 3: Let's construct the dependence of the fractal dimension of the image on the range of gradations of brightness.

The smaller the size of the test image, i.e. "Windows", the faster the value of the fractal dimension tends to a value of 2.5.

The error modulus $\delta D$ of determining the fractal dimension in the "window" depends on the brightness range $\Delta I$ as follows:

$$
\delta D=|2.5-B(\Delta I)|
$$

Fig. 4 shows that at small brightness ranges, i.e. - this is a low contrast image, it is more reasonable to use "windows" not exceeding $17 \times 17$ pixels. 


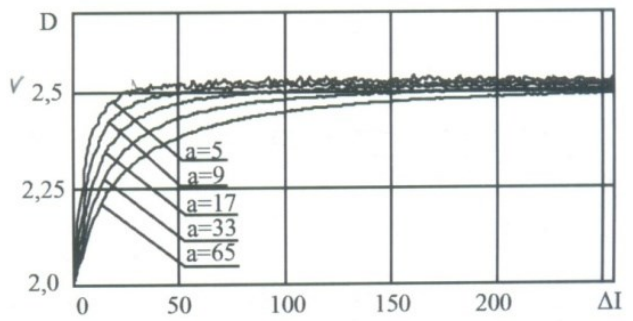

Fig. 4. The dependence of the fractal dimension with different sizes of the "window" on the range of brightness gradations.

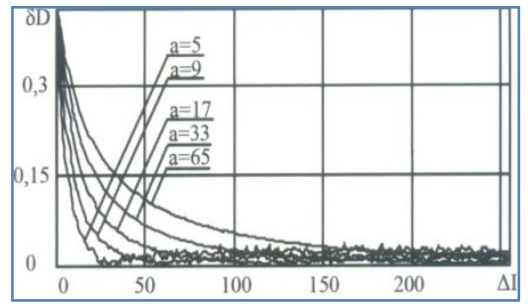

Fig. 5. Connection of the error of determining the fractal dimension of the range of brightness gradations

Table 1 shows the field values of the fractal dimension (FFD) when processing the original image with a "sliding" window of various sizes $(a \times a), s-$ window moving step.

\begin{tabular}{|l|l|l|l|l|}
\hline \multirow{2}{*}{$\begin{array}{l}\text { Window moving } \\
\text { step }\end{array}$} & \multicolumn{4}{|l|}{ Window size } \\
\cline { 2 - 5 } & $5 \times 5$ & $9 \times 9$ & $17 \times 17$ & $33 \times 33$ \\
\hline $\mathrm{s}=1$ & 896 & 892 & 884 & 868 \\
\hline $\mathrm{s}=\mathrm{a}$ & 180 & 100 & 53 & 27 \\
\hline
\end{tabular}

Now let's conduct a number of further studies in terms of image texture.

From fig. $6 \mathrm{a}$ we can see that the littering texture is visible, as well as the texture of the forest has regrularity. Some coatings by littering, as well as forest coverings, have the same regularity in all directions, others - in some directions are much larger than in others. This can be observed in the correlation images of $\mathrm{C}$ textures (b), which have bands responsible for the preferred direction of regularity. The elements of $C(x, y)$ show the correlation coefficient of twodimensional signals from a point (1.1) of constant length $\mathrm{d}$, smaller than the size of the original image, and the shifted signals by $\mathrm{x}$ to the right and $\mathrm{y}$ down. So CML histograms (c) along vertical, horizontal and any other sections for forests are approximately the same in terms of fractal characteristics, and for littering, more regular along certain directions. In this regard, the fractal signs of littering are "more mobile" than forests.

WDS is an object of random but regular form and a CML; therefore it can be detected by fractal signs. Fractals are objects that have self-similarity and are measured by different parameters: self-similarity density, fractal dimension and its field, accuracy of determination (construction), etc. 

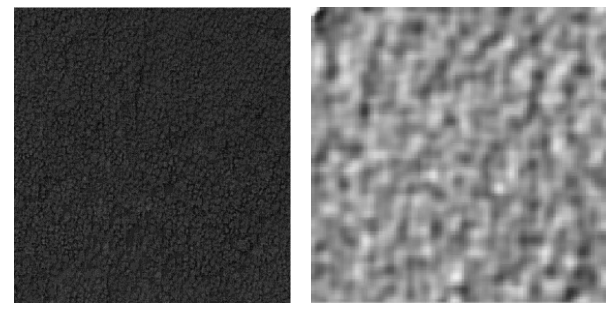

(a1)

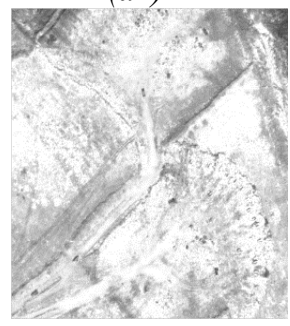

(b1)

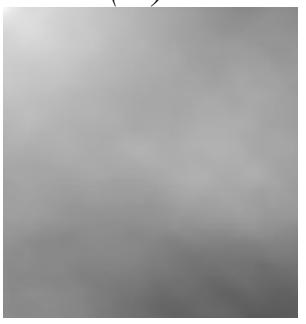

(a2)

(b2)

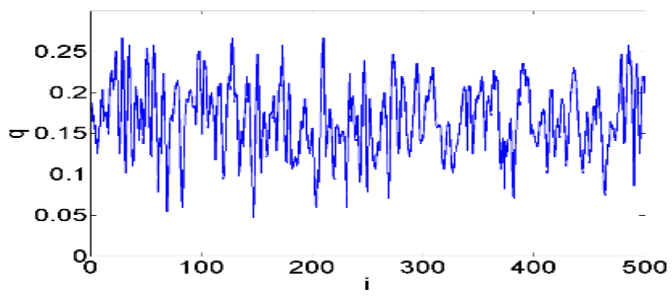

(c1)

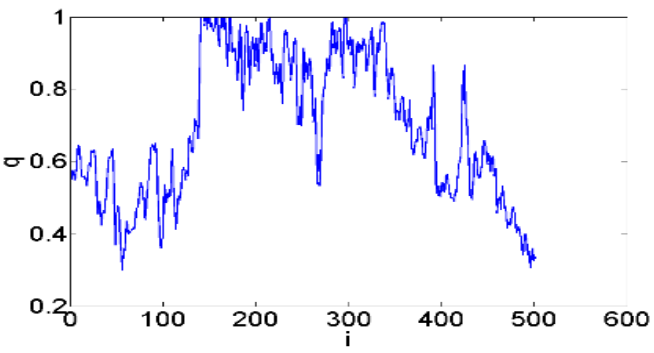

(c2)

Fig. 6. Texture patterns (blue channel) (a), patterns of correlation images (b), values of the spectral brightness coefficient (CML) in one of the sections;

1) forest, 2) littering

Color has little effect on the value of the fractal dimension, so its estimate can be given by one channel. Some of the "fractal" trash coverings have the same texture as the forest (see fig. 7a), and most of them refer to the typical trash texture, but with a difference in the composition of colors. So areas covered by littering at different times of the year and time of day have close fractal dimensions.

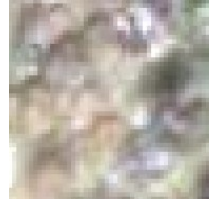

(a)

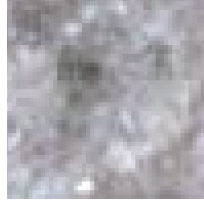

(b)

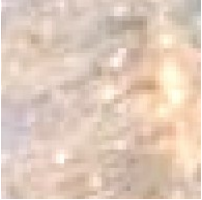

(c)

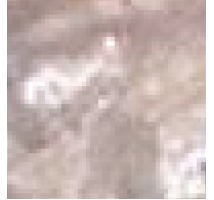

(d)

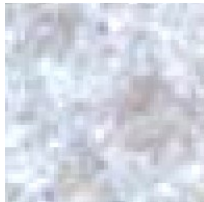

(e)

Fig. 7. Littering textures of different chromaticity, but close in fractal dimension: a) $f=1.9249$; b) $f=1.8729$; c) $f=2.0952$; d) $f=2.0426$; e) $f=2.0726$

The fractal dimension $f$ is calculated as the slope of the regression line of the reports $\mathrm{y}(x)$, where: 


$$
x=\ln \frac{1}{r}, y=\ln N, \quad N(r)=\sum_{k=1}^{l} \frac{b_{k}}{a_{k}}
$$

$a_{k}$ and $b_{k}$ - the minimum and maximum CML of the k-th cube of the original image when it is cut into cubes of size r. Fig. 8 shows an example of fractal calculation dimensions for the standard in fig. 7 a.

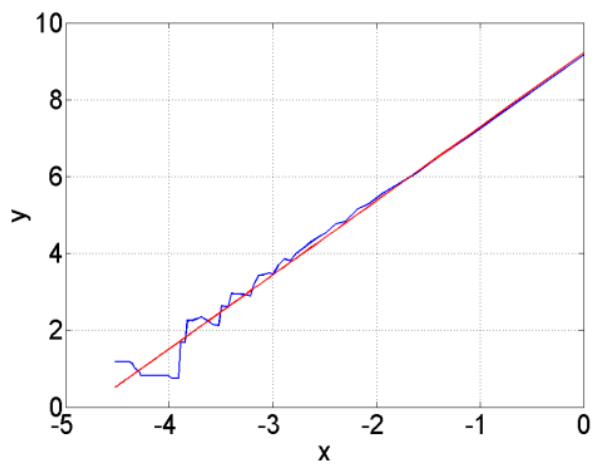

Fig. 8. Calculation of the fractal dimension

Another formula for calculating $N$ :

$$
N(r)=\sum_{k=1}^{l}\left(b_{k}-a_{k}\right)
$$

As shown by calculations using model (3), the fractal dimensions of the littering plots lie in the range of $1.85 \div 2.1\left(f_{\min }=1.85, f_{\max }=2.1\right)$ and turn out to be close to the dimension of the ideal uniform distribution of 2.5 (see Fig. 7). For comparison, the dimension of the forest massif, which, unlike littering, has an ordered cell structure, according to this model, is $1.5 \div 1.6$. Turns, spatial resolution, image size, scale of its increase or decrease do not affect the fractal dimension (Fig. 9)

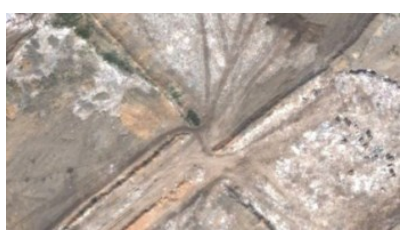

(a)

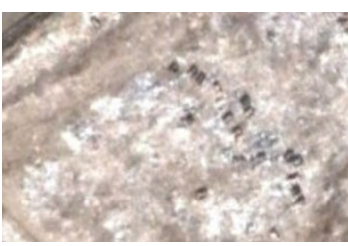

(b)

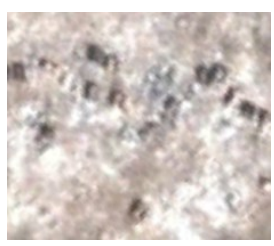

(c)

Fig. 9. Textures of littering: a) a large area $(f=1.8)$; b) with an increase in its area $(f=1.8405)$; c) at higher magnification and rotation $(f=1.8148)$ [Google Earth] 
The trash areas are detected by the values of the fractal dimensions, according to the scheme: 1) the original image is scanned with a square window with a side-by-side focus on each pixel; 2) the fractal dimension is calculated by the model (3) in a square neighborhood $r$ of each point, i.e. the field of fractal dimensions is calculated; 3 ) the threshold filtering method is used to allocate a detection area with fractal dimensions for littering $1.85 \div 2.1$.

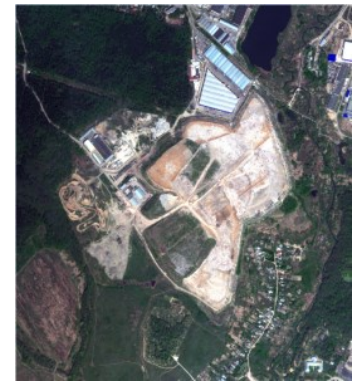

(a)

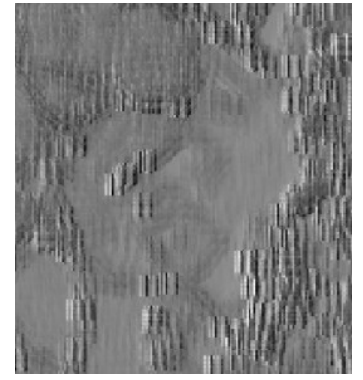

(b)

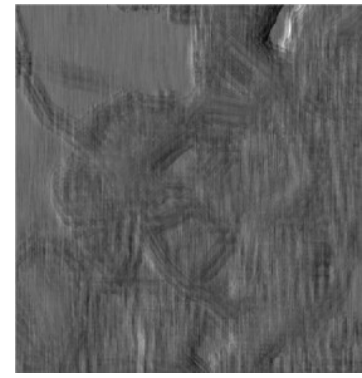

(c)

Fig. 10. Original image (a); field of fractal dimensions by model: b) (1), c) (2)

Fig. 10 shows an example of normalized fields of fractal dimensions for the image (a), calculated by models (1) and (2).

More precisely, WDS are detected by the dependences $f(q)$, where $f$ is the fractal dimensions of binary images $J(q)$ obtained from $I$ the original $I$ (texture image) by threshold filtering on the lower level of the CML q. The functions $f(q)$ exponentially decreases from the maximum fmax to the minimum fmin characteristic of this texture I. For the surfaces of different texture patterns, these dependences differ (Fig. 11).

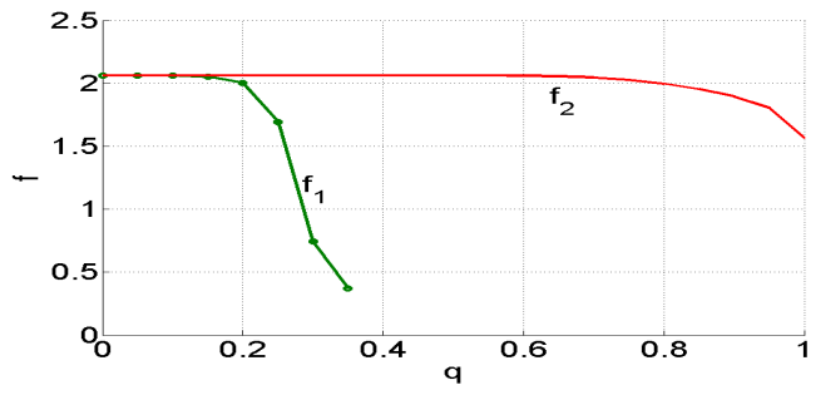

Fig. 11. Dependencies of the fractal dimensions $f$ on the threshold CML $q$ for covering: $f_{1}$ is a forest, $f_{2}$ is littering 
The proposed method of fractals will allow overcoming the problems of processing the SPE. This is due primarily to the incompleteness of knowledge about signals, non-stationarity, non-Markovism, noise singularity based on preliminary information about the spatial scales of the detected signals.

\section{Conclusion}

One of the leading directions in the analysis of aerospace monitoring data is the development of the theory of processing digital multispectral and hyperspectral images of the Earth and the atmospheric surface layer to solve the problem of automatic (without human participation) recognition of useful signals localized in time and space randomly in the background of additive and multiplicative noise. The experiment leads to the following conclusions:

- FFD can be used to analyze SI with a WDS image.

- The size of the "window" depends on the range of brightness values of the image.

- Performing detailed segmentation of the image requires the presence of "sliding windows".

. The speed of building a FFD is increased by using "jumping windows".

Thus theresearches of image texture and the construction of FFD were held.

\section{References}

1. Arunyants, G. G., and Kazaryan M. L. Class of systems of orthogonal Haar functions based on modified Rademacher complex functions // Vladikavkaz Mathematical Journal. 2005, 7, 1, 16-24.

2. Kazaryan, M. L., Richter A. A., Shakhramanyan M. A., and Nedkov R. Space monitoring of solid household waste and industrial waste disposal facilities (MSW and software): theoretical, methodological and socio-economic aspects: monograph / - M .: INFRA-M, 2019, 280 p. (Scientific thought).

3. Schovengerdt, R. A. Remote sensing. Methods and models of image processing Moscow: Technosphere, 2010. $560 \mathrm{p}$.

4. Mandelbrot Century. Century. The Fractal Geometry of Nature. W., H. Freeman, New York, 1983. $468 \mathrm{p}$.

5. Chen, S., Keller J., Crownover M. On the Calculation of Fractal Features from Images. IEEE Transactions on Pattern Analysis and Machine Intelligence. 1993, 15, JSFO $10,1087-90$.

6. De Cola, L. Fractal analysis of a classified Landsat scene. Photogrammetric Engineering and Remote Sensing. 1989, 55, 5, 601-10.

7. Stein, M. C. Fractal image-models and object detection. Visual Communications and Image Processing 11, Proceedings of SPIE. 1987, 845, 293-300.

8. Peli, T. Multiscale fractal theory and object characterization. Journal of the Optical Society of America. 1990, 7, 6, 1101-12. 
9. Pentland, A. R. Shading into Texture. Artificial Intelligence. 1986, 29, 147170.

10. Carr, J. R., Numerical analysis for the geological sciences: Prentice Hall, 1995.

11. Kazaryan, M., Shahramanian M., Voronin V. "The automated space monitoring system of waste disposal sites," Proc. SPIE 10793, Remote Sensing Technologies and Applications for Urban Environments III, 1079318 (9 October 2018); DOI:10.1117 / 12.2500059 .

12. Sun, W. Fractal analysis of remotely sensed images: A review of methods and applications. W. Sun, G. Xu, P. Gong, S. Liang. Int. Remote Sens. 2006, 27, 22, 4963-90.

13. Pratt, E. Digital image processing, Book. 1.: Trans. from English M .: Mir, 1982. 312 p.

14. Methods of computer image processing / Ed. Soifer V.A. 2nd ed., Corr.

15. Yaroslavsky, L. P. Digital signal processing in optics and holography: Introduction to digital optics / M .: Radio and Communications, 1987, 296 p.

16. Tikhonov, A. N., Arsenin V. Ya. Methods for solving incorrect problems. 2nd ed. M .: Science: Main edition of the physical and mathematical literature, $1979.285 \mathrm{p}$.

17. Fursov, V. A. Analysis of the accuracy and construction of identification algorithms for a small number of observations. Izv. Academy of Sciences of the USSR, Techn. Cybernetics, 1991, 6.

18. Haralick, R. M. Statistical and structural approaches to texture. Proceedings of the IEEE, 1979, 67, 5, 768-804.

19. Duda, R., Hart P. Pattern Recognition and Scene Analysis: Trans. from English, M.: Mir, 1976, $512 \mathrm{p}$.

20. Zhuravlev, Yu. I. On the algebraic approach to solving problems of recognition or classification // Problems of Cybernetics, "Science", Moscow, vol. 33, 5-68 (1978)

21. Lapko, A. V. Non-parametric methods of classification and their application // Novosibirsk: IN Science. 1993. 152 p.

22. Bel'chanskiy, G., V. A. Yeremeyev, I. N. Mordvintsev, and N. G. Platonov. Comparative Analysis of the Sea of Ice Concentrations Determined Using the Okean-O1, SSM / I, and Radarsat Satellite Data. Mapping Sciences and Remote Sensing. V.H.Winston \& Son, Inc. / Bellwether Publishing Ltd. Columbia MD. USA. 2002, 39, 1, 56-65.

23. Sergeev, V. V. Image processing using the Hilbert-Peano scan // Novosibirsk: Nauka. 1984.

24. Hramov, A. G. The method of the field of directions in the analysis and interpretation of diagnostic images // Authors. bottom. Samara. 2006. 32 p.

25. Potapov, A. A, Fractals in radiophysics and radiolocation: Sampling topology // M .: University book. 2005. 848 p.

26. Lavrova, O. Yu., Loupian E. A., Mityagina M. I., Uvarov I. A., Bocharova T. Yu. Multi-User Guidelines for Satellite Remote Sensing Data // Bollettino di Geofisica teorica ed applicata. An International Journal of Earth Sciences. 2013, 54, 146-47.

27. Loupian, E. A, Mazurov A. A, Flitman E. V., Ershov D. V., Korovin G. N., Novik V. P., Abushenko N. A., Altyntsev D. A., Koshelev V. V., Tashchilin S. A., Tatarnikov A.V., Csiszar I., Loboda T., Sukhinin A. I., Ponomarev E. I., fonin S. V., Belov V.V., Matvienko G. G. Satellite Monitoring for Global Change. 2006, $11,1,113-45$. 


\title{
ПРОВЕДЕНИЕ МАТЕМАТИЧЕСКИХ - СИСТЕМНЫХ ИССЛЕДОВАНИЙ С ПРИВЛЕЧЕНИЕМ ФРАКТАЛОВ ПРИ АВТОМАТИЗАЦИИ ОБРАБОТКИ КОСМИЧЕСКИХ СИСТЕМ НАБЛЮДЕНИЯ ПО РАСПОЗНАВАНИЮ ОБЬЕКТОВ ЗАХОРОНЕНИЯ ОТХОДОВ
}

\begin{abstract}
М. Казарян
Аннотация

В статье дан анализ факторов, стимулирующих исследования и разработку автоматизированной системы по обработке и анализу данных космических снимков. В частности, в качестве приложения рассматривается исследование космических снимков на наличие объектов захоронения отходов. Данная проблема представляет особый интерес при проведении научных изысканий. Разработка автоматизированных систем управления (АСУ) в области распознавания космических снимков (КС) характеризуется, прежде всего, интересуемым подходом, т.е. для какой цели и для решения какой именно задачи предметной области создается система. Очевидно, система это совокупность определенных информационных технологий и главным фактором при разработке автоматизированной системы есть и остается изучение особенностей при создании соответствующей информационной системы (ИТ). В статье предлагается методологический подход к обработке и анализу данных КС. Подход основывается на концептуальной идее фрактальных множеств. Это повышает обнаружение аномальных сигналов в сложной фоноцелевой обстановке. Проводится апробация по обнаружению ОЗО в условиях малого отношения сигнала к шуму.
\end{abstract}

